
$\mathrm{Gu}^{*}$

\title{
Mechanical characterizations of braided composite stents made of helical polyethylene terephthalate strips and NiTi wires
}

https://doi.org/10.1515/ntrev-2019-0016

Received Mar 04, 2019; accepted Apr 11, 2019
Abstract: The novel braided composite stent (BCS), woven with both nitinol wires and polyethylene terephthalate (PET) strips, were characterized and compared with the braided nitinol stent in the same weaving pattern. Finite element models simulating the stent compression and bending were developed to quantify its radial strength and longitudinal flexibility. The interaction between the nitinol wires and the PET strips were also delineated. Results showed that the PET strips enforced more constrains on the BCS and thus enhance its radial strength especially at a larger compression load. The longitudinal flexibility of the BCS was less sensitive to the presence of the PET strips. This work suggested that the novel design of the BCS could acquire the advantage of a covered stent without compromising its mechanical performance. The fundamental understanding of the braided composite stent will facilitate a better device design.

Keywords: Braided composite stent, wire interaction, radial strength, flexibility, finite element method

\footnotetext{
^Corresponding Author: Meiwen An: Institute of Biomedical Engineering, College of Biomedical Engineering, Taiyuan University of Technology, Taiyuan 030024, China; Email: meiwen@tyut.edu.cn *Corresponding Author: Linxia Gu: Department of Biomedical and Chemical Engineering and Sciences, Florida Institute of Technology, Melbourne, FL 32901-6975, United States of America; Email: gul@fit.edu

Qingli Zheng: Institute of Biomedical Engineering, College of Biomedical Engineering, Taiyuan University of Technology, Taiyuan 030024, China

Pengfei Dong: Department of Mechanical \& Materials Engineering, University of Nebraska, Lincoln, NE 68588, United States of America Qingli Zheng and Pengfei Dong contributed equally to this paper Zhiqiang Li: Institute of Applied Mechanics, College of Mechanical and Vehicle Engineering Taiyuan University of Technology, Taiyuan 030024, China

Xinwei Han: Department of Interventional Radiology, The First Affiliated Hospital of Zhengzhou University, Henan, China
}

\section{Introduction}

Braided nitinol stents (BNS) are increasingly popular in treating arterial occlusions, especially peripheral arterial disease [1]. Compared to the laser-cut stents, the BNS tend to have a reduced radial strength and better flexibility [2]. The radial strength of the stent was essential to keep the narrowed vessel open [3]. The flexibility of the stent was desired to move along or scaffold a curved vessel. Despite the demonstrated acute outcomes of a stented vessel, the long-term patency of the BNS remains a challenge due to tissue ingrowth through the mesh structure of stents [4].

Covered stents, i.e., stents covered by a fabric or graft material such as polytetrafluoroethylene or polyethylene terephthalate (PET), were designed to mitigate the tissue ingrowth as well as to seal the vessel perforation [5]. However, compared to a bare metallic stent, the covered stent is harder to maneuver through tortuous anatomy without the risk of kinking and migration $[6,7]$. This led to undesired vessel patency as well as tissue damage and increase incidence of limb thrombosis [8]. Recently a braided composite stent (BCS) woven using PET strips and nitinol (NiTi) wires was proposed to improve the radial strength as well as the flexibility of a BNS [9]. Considering the minimal porosity of the BCS, it drove us to consider the BCS as a potential alternative to the covered stent.

Finite element method (FEM) has proven to be an effective tool for the characterization of the stent performance and its underlying mechanism. It could visualize the mechanics virtually anywhere in the computational domain, a feature that is not affected by experimental techniques. Kim et al. developed a 3D model to quantify the mechanical behavior of braided stents validated by experiments [10]. The FEM results from Zhao et al. showed that the braided stent had a relative small radial strength than

Changchun Zhou: National Engineering Research Center for Biomaterials, Sichuan University, 610064, Chengdu, China 
the welded or laser cut ones [2]. De Beule et al. adopted a virtual optimization algorithm to design a braided stent with less foreshortening while maintaining the radial stiffness [11]. However, no FEM studies were related to the BCS.

In this work, the mechanical behaviors of the BCS were investigated through FEM. Three-dimensional models of the BCS and the corresponding BNS in the same weaving pattern were developed to evaluate and compare their radial strength and flexibility. The BCS model was validated by the published experimental results [9]. The effects of PET strips as well as the interaction between PET strips and NiTi wires were delineated. Quantitative evaluation of the mechanical performance of the BCS and its underlying mechanisms could facilitate an optimal design of the stent.

\section{Materials and methods}

Three-dimensional models of the BCS as well as its counterpart BNS were constructed as shown in Figure 1. Both BCS and BNS have an outer diameter of $7 \mathrm{~mm}$, length of $15 \mathrm{~mm}$, and a braided angle $\beta$ as $65^{\circ}$. The braided angle is the angle between the helical wire and the axial direction of the stent. The BNS was braided by 32 strands of helical NiTi wires with a wire diameter of $0.2 \mathrm{~mm}$. The BCS was braided by 8 NiTi wires and 24 PET strips. The thickness of the PET strip was $0.12 \mathrm{~mm}$ [12]. The width of the PET strip (w) was $0.57 \mathrm{~mm}$, which could be calculated as following:

$$
w=L \cos (\beta)=\frac{\pi D}{16} \cos (\beta)
$$

where $L$ is the distance of the two crossover points along the circumferential direction (Figure 1a).

The superelastic constitutive model of NiTi wires was implemented through a built-in Abaqus User Material Subroutine (VUMAT) and the material parameters were adopted from literature [13]. The PET strip was characterized by an elastoplastic material model [14]. Both material parameters were summarized in Table 1. Following the mesh-sensitivity study, each NiTi wire was meshed with 240 two-node linear beam elements (B31). The PET strips were meshed with 23,040 reduced 4-node doubly curved shell elements (S4R).

Compression tests were conducted in silico to quantify the radial strength of the stent (Figure 1c). Specifically, the middle section of the stent was vertically compressed by a rigid cylinder of $5 \mathrm{~mm}$ in diameter. The displacement control of $3.5 \mathrm{~mm}$, i.e., $50 \%$ of the outer diameter of the stent, was enforced on the cylinder [15]. The reaction force of the cylinder, i.e., load applied on the stent, positively corre-

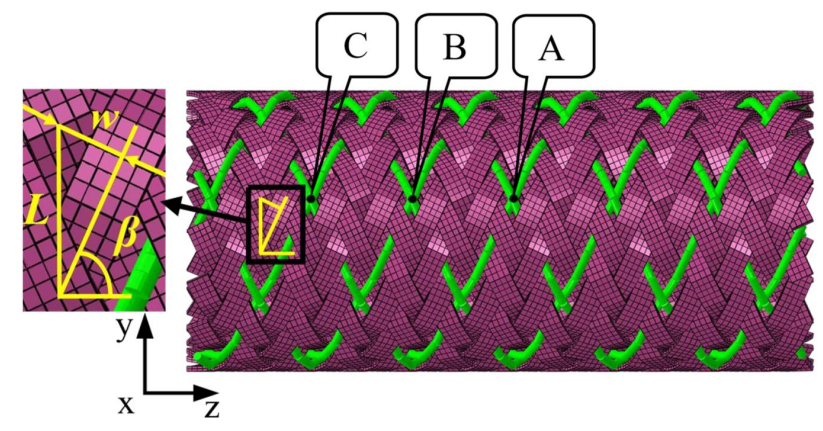

(a)

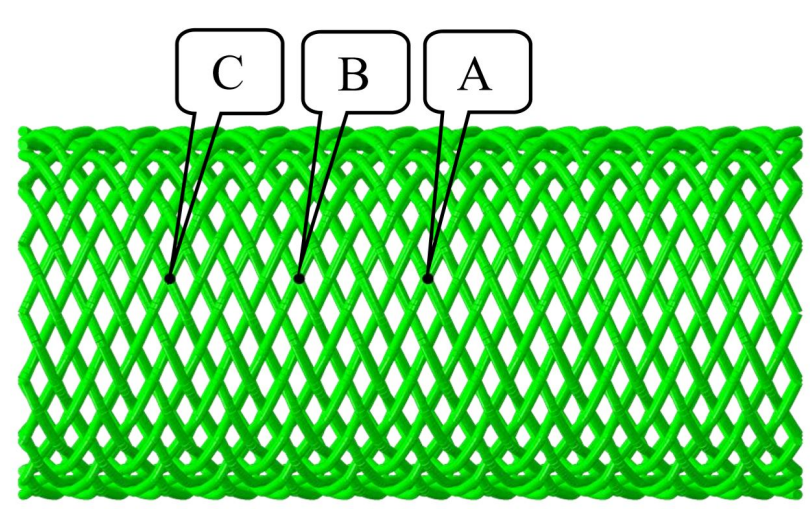

(b)

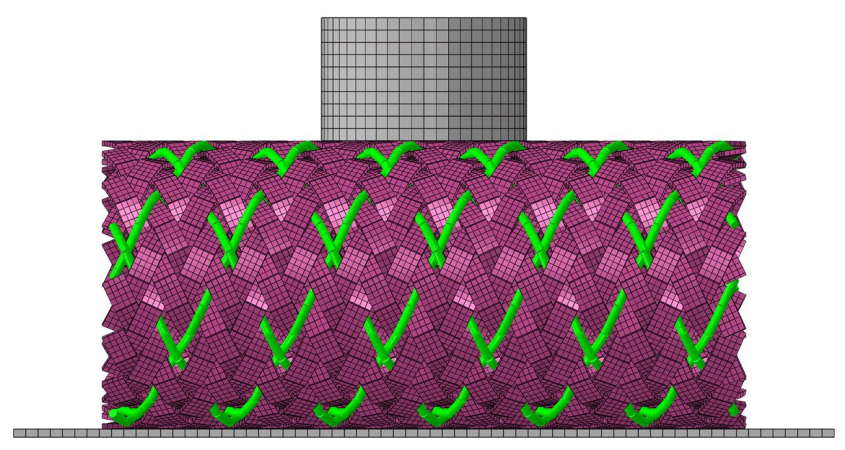

(c)

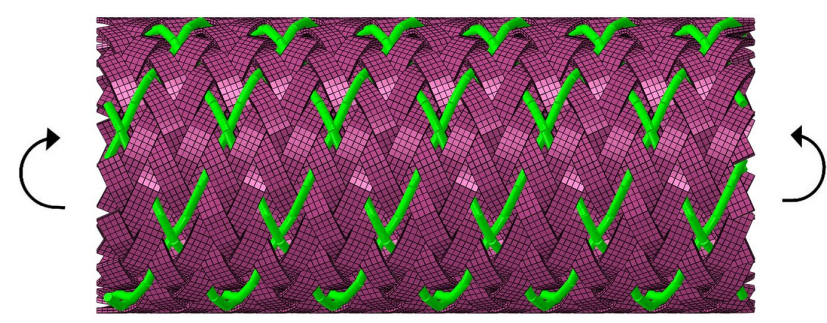

(d)

Figure 1: Three-dimensional models of (a) braided composite stent and (b) braided nitinol stents, (c) compression test, (d) bending test. 
Table 1: Material properties of nitinol and polyethylene terephthalate.

\begin{tabular}{lc}
\hline Nitinol & 50000 \\
Austenite elasticity $\mathrm{E}_{A}(\mathrm{MPa})$ & 37000 \\
Martensite elasticity $\mathrm{E}_{M}(\mathrm{MPa})$ & 400 \\
Start of transformation loading $\sigma \mathrm{S} \mathrm{L}(\mathrm{MPa})$ & 650 \\
End of transformation loading $\sigma \mathrm{E} \mathrm{L}(\mathrm{MPa})$ & 350 \\
Start of transformation unloading $\sigma \mathrm{S} U(\mathrm{MPa})$ & 80 \\
End of transformation unloading $\sigma \mathrm{E} \mathrm{U}(\mathrm{MPa})$ & 0.055 \\
Volumetric transformation strain $\epsilon \mathrm{L} V$ & \\
\hline Polyethylene terephthalate $(\mathrm{PET})$ & 3.5 \\
Young's modulus $\mathrm{E}(\mathrm{GPa})$ & 0.4 \\
Poisson ratio $v$ & 60 \\
Yield stress $\sigma_{s}(\mathrm{MPa})$ & 70 \\
Tensile stress $\sigma_{t}(\mathrm{MPa})$ & \\
\hline
\end{tabular}

lated with the radial strength of the stent, was monitored during the load and unload process.

The stent flexibility was quantified by the pure bending tests (Figure 1d). Both ends of the stent were fixed to a rigid plane with rotations around the $\mathrm{X}$ axis in opposite directions until the angle between two end surfaces reached at $60^{\circ}$ [16]. The corresponding load, i.e., bending moment, was recorded.

A general contact algorithm was considered among all contact surfaces with friction coefficients of 0.3. The kinetic energy of each stent was kept under $5 \%$ of the internal energy to avoid the inertia effect. Abaqus/Explicit solver (Dassault Systèmes Simulia Corp.) was used due to its demonstrated capacity for complex contact problems.

\section{Results}

The radial strength of the BCS was compared with the published data as illustrated in Figure 2. The percentage deformation was estimated with reference to the original outer diameter of the stent. It is clear that our computational results matched well with the experiment (Model B2) [9].

The compression induced stress and strain distributions in both BCS and BNS were shown in Figure 3. The peak von Mises stress occurred on the nitinol wires for both stents. It was $581 \mathrm{MPa}$ and $600 \mathrm{MPa}$ for the BCS and BNS, respectively. Both values fall in the range of transformation loading (400 to $650 \mathrm{MPa}$ ) as defined in Table 1. It indicated that the deformation of nitinol wires reached at the plateau of phase transformation between austenite and martensite. The maximum tensile strain (SDV24) of the nitinol wires was $7.06 \%$ in BCS and 7.12\% in BNS. Both were

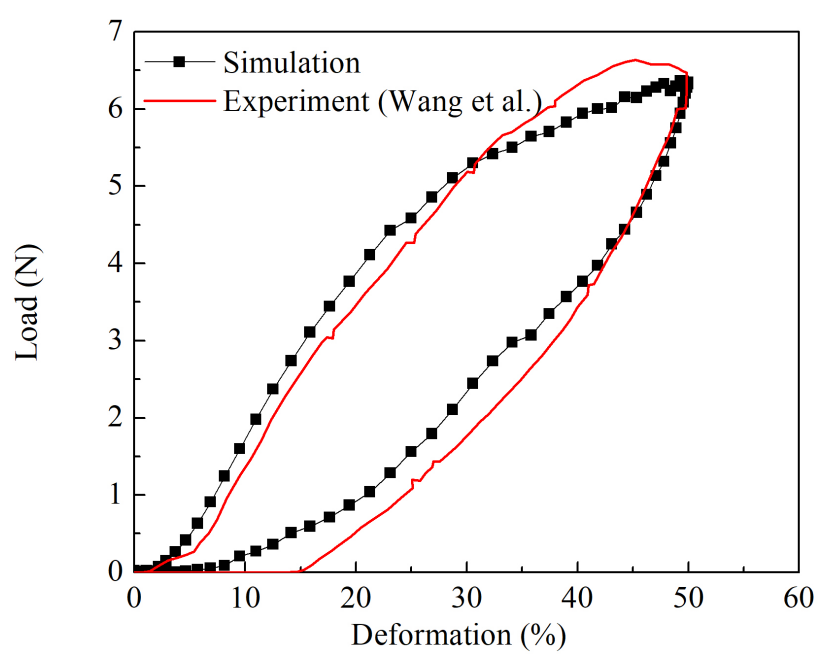

Figure 2: Simulation of the braided composite stent compression in compared to experimental results.

below the elastic limit of $8 \%$, indicating that nitinol wires could fully recover back to its original configuration following the compression. The peak von Mises stress and strain in the BCS strips was $60.6 \mathrm{Mpa}$ and $11.8 \%$, respectively. In addition, the stress or strain distribution patterns of BCS were different with the ones of BNS. Specifically, the stress and strain of the BNS concentrated beneath the cylinder surface (Figure 3c), while the stress and strain of the BCS concentrated around its lateral region (Figure 3a\&b). The braiding angle at the stress concentration area of the BCS decreases from the original $65^{\circ}$ to $44.9^{\circ}$ while it decreases further to $35.2^{\circ}$ for the BNS (Figure $3 \mathrm{a} \& \mathrm{c}$ ). This indicated the wire rotation and slippage during the loading.

The load-deformation diagrams of both BCS and BNS were depicted in Figure 4. It was clear that the BCS and BNS demonstrated a similar compressive load at lower deformation, i.e., less than $20 \%$. Thereafter, the compressive load in BNS exhibited a plateau at approximately 4.2 $\mathrm{N}$, while the compressive load of the BCS continually increased up to $6.4 \mathrm{~N}$.

The slippage was estimated by monitoring the relative displacement at three representative wire intersection locations (A, B, and C, from center to the end of stent, as labeled in Figure 1). The relative displacements of these three locations subject to compression were depicted in Figure 5. It was clear that the stent deformation positively correlated with the relative deformation of wires. At the compression deformation of $50 \%$, the relative displacements were $0.19 \mathrm{~mm}, 0.34 \mathrm{~mm}$ and $0.16 \mathrm{~mm}$ for the location $\mathrm{A}, \mathrm{B}$ and $\mathrm{C}$ of the BCS, respectively. For the BNS, they were $0.13 \mathrm{~mm}, 0.56 \mathrm{~mm}$ and $0.62 \mathrm{~mm}$, respectively. The results showed that the BCS showed a more uniform and smaller 


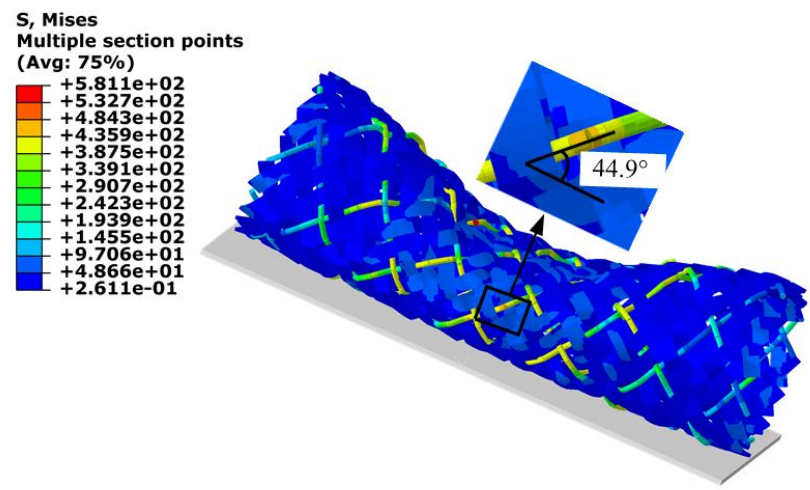

SDV24

Rel. radius $=1.0000$, Angle $=\mathbf{- 9 0 . 0 0 0 0}$

(Avg: 75\%)

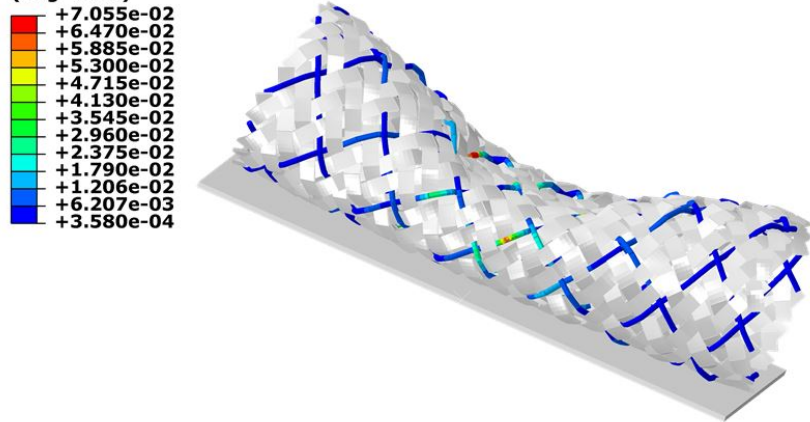

(a)

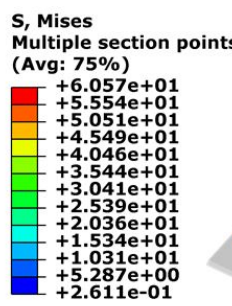

Multiple sect

(Avg: 75\%)

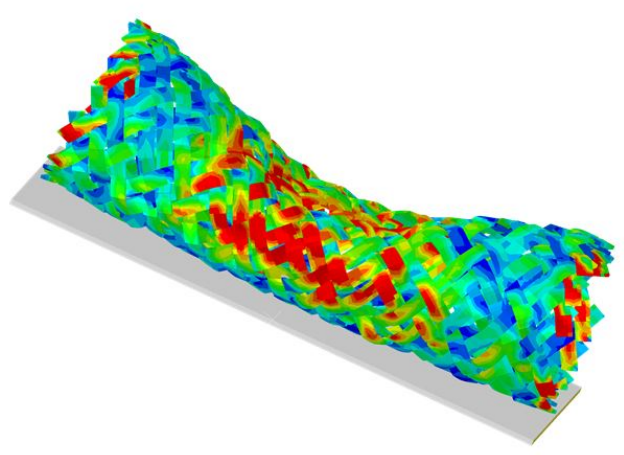

(b)

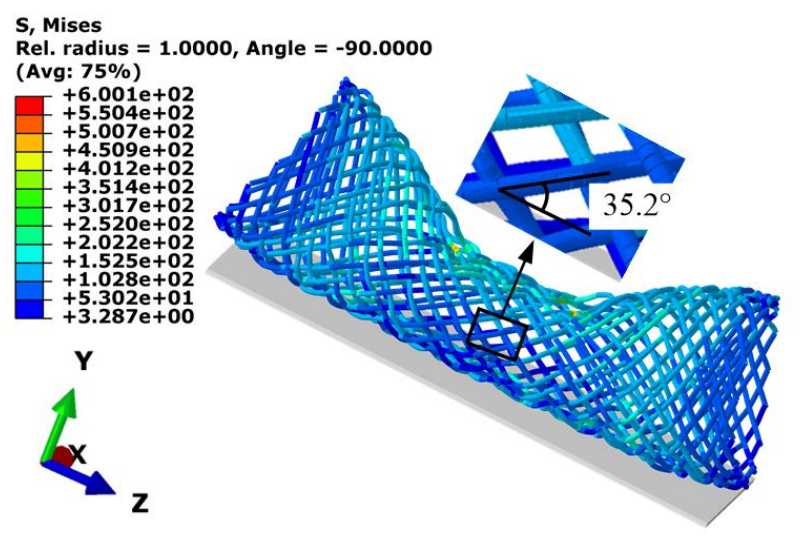

SDV24

Rel. radius $=1.0000$, Angle $=\mathbf{- 9 0 . 0 0 0 0}$

(Avg: 75\%)
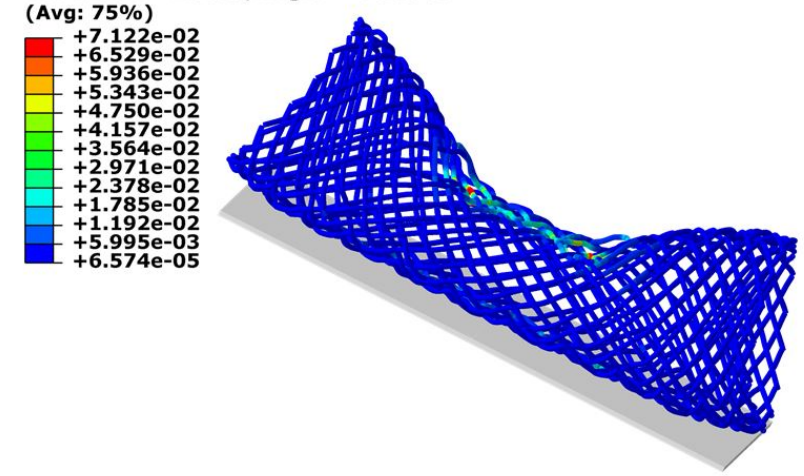

(c)

Figure 3: Compression induced stress (left) and strain (right) distributions for (a) nitinol wires in braided composite stent; (b) PET strips of braided composite stent; and (c) braided nitinol stents. 


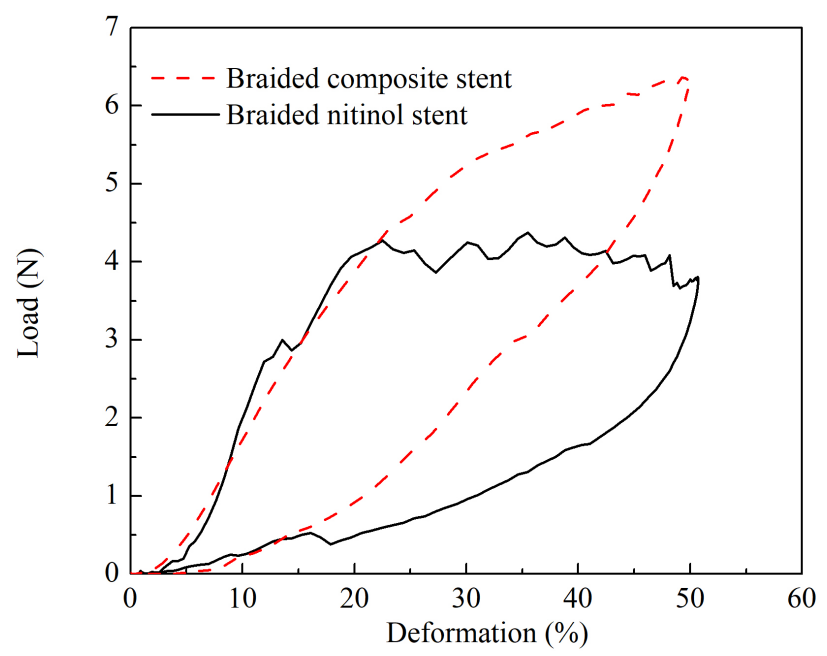

Figure 4: The radial compression of braided composite stent and braided nitinol stent

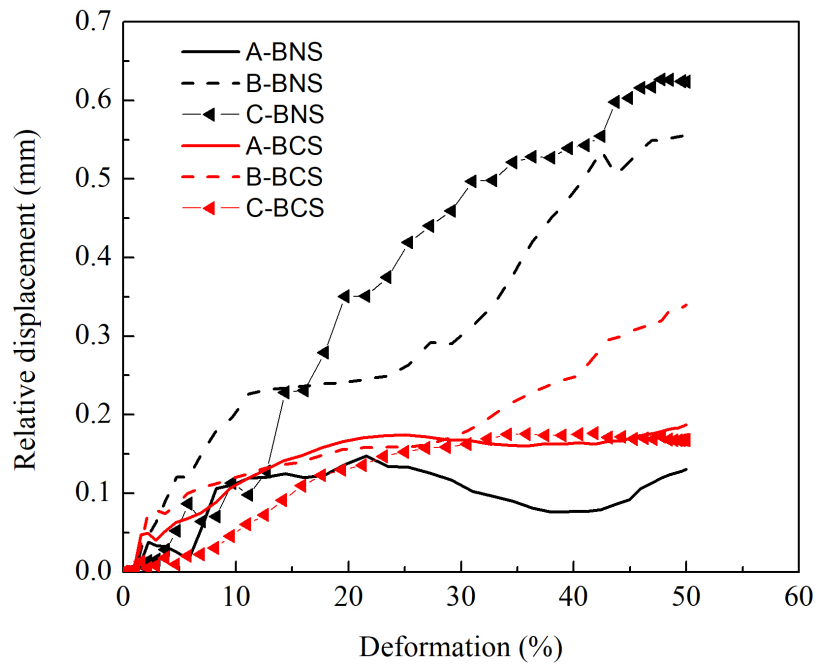

Figure 5: Relative displacement of two points between intersections of NITI wires of braided composite stent and braided nitinol stent under radial compression.

relative displacement than the BNS, the larger relative displacement in BNS may contribute to the unstable radial force at the larger deformation.

The stress distribution of the stents during the bending test were shown in Figure 6 . At the bending angle of $37^{\circ}$, the peak value of von Mises stress in BCS was $294 \mathrm{MPa}$, which was larger than that in the BNS of $167 \mathrm{MPa}$, the same trend was found at the bending angle of $60^{\circ}$, which was $404 \mathrm{MPa}$ in BCS and $260 \mathrm{MPa}$ in BNS. The peak value of von Mises stress in two stents were both located in the nitinol wires. The bending moment for each stent during the bending test was shown in Figure 7. The BCS demonstrated a relative larger bending moment than the BNS at a bend- ing angle larger than $37^{\circ}$. At a bending angle of $60^{\circ}$, the bending moment of the BCS was $24 \%$ larger than that of the BNS.

\section{Discussions}

In this work, the design of BCS was systematically characterized in terms of radial strength and longitudinal flexibility. The mechanical behaviors of the BCS were compared with the BNS with the same weaving pattern. The major difference lies in the replacement of 24 strands of NiTi wires by the wider PET strips. The BCS possessed the advantage of covered stent without compromising their mechanical performance. The BCS provided a direct barrier to the tissue underneath. Moreover, the radial strength of the BCS was larger than the BNS especially at the larger radial deformation. The bending moment was smaller than the BNS when the bending angle was less than $37^{\circ}$ and much less than the reported ones of the covered stent. When the polyurethane or silicone covered stents were subjected to a bending angle of $30^{\circ}$, the required forces were $11 \%$ and $44 \%$, respectively, more than the BNS [17]. While at a bending angle of $30^{\circ}$, the bending moment of the BCS was 9.3\% smaller than that of the BNS.

The radial strength of the stent, i.e., the capability to scaffold the stenotic lumen, is the first consideration of the stent conceptual design $[18,19]$. The BCS and BNS showed the same radial strength when the deformation is less than $20 \%$. This seems different from the documented experiment [9], which stated that the radial strength of the BCS was much larger than the BNS. This could be explained by the different braided angle for the BCS and BNS. Specifically, it was $65^{\circ}$ for BCS and $30^{\circ}$ for BNS. The braided stent with a larger braided angle resulted in a higher radial strength [10]. As the deformation exceeded $20 \%$, the radial strength of the BNS reached a plaque of $4.2 \mathrm{~N}$, while the BCS kept increasing up to $6.4 \mathrm{~N}$. The different radial strength of the BCS and BNS at a large deformation could be attributed to the interaction between the PET strips and NiTi wires. In BNS, there existed the relative rotation and slippage between NiTi wires. However, the presence of wide PET stripes in BCS constrained the relative rotation and slippage of the NiTi wires. Our compression tests have shown that the alteration in the braided angle of the BNS was $48 \%$ larger than that of the BCS. This was also reflected in the relative larger displacement of the BNS than that of the BCS. A large relative movement in the woven wires was associate with the smaller radial strength and a larger longitudinal flexibility. 

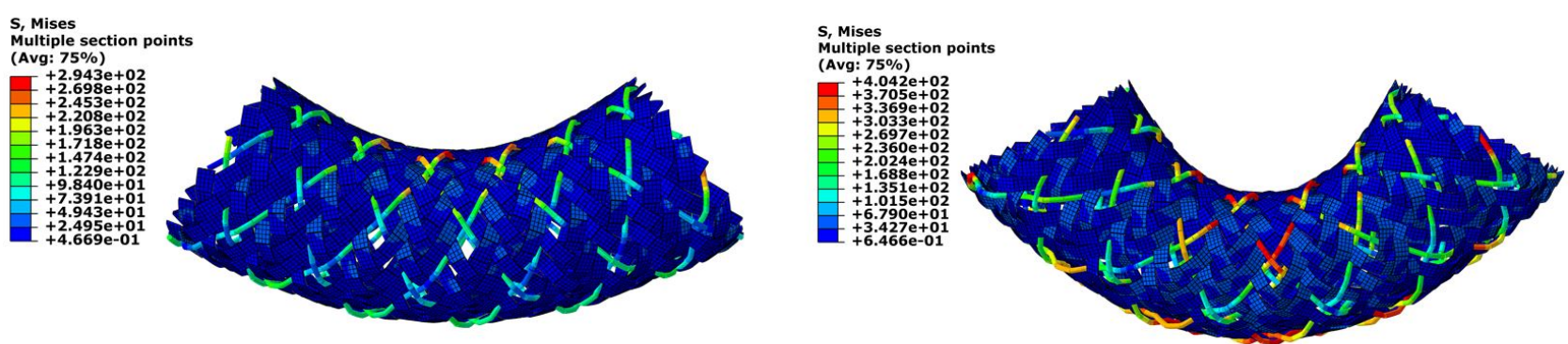

(a) Braided composite stent
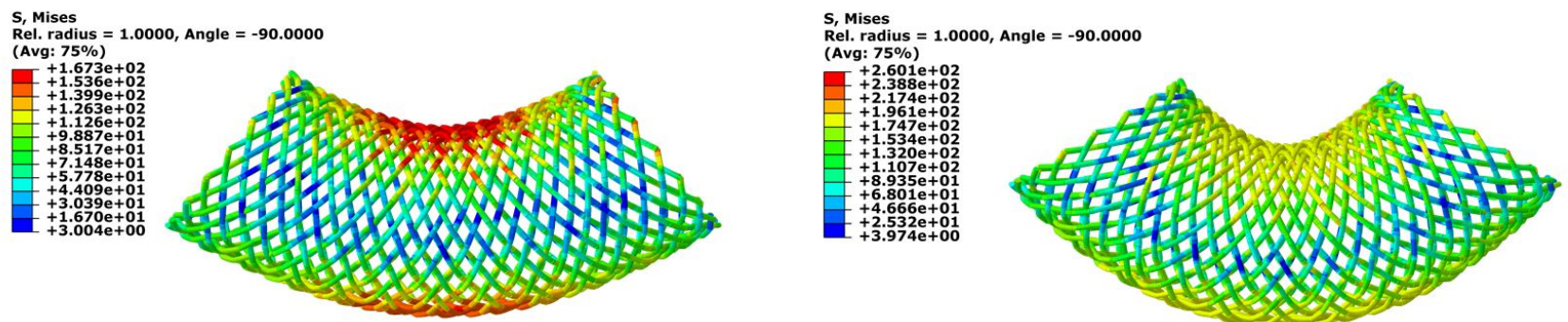

(b) Braided nitinol stent

Figure 6: Bending behaviors of braided composite stent and braided nitinol stent at the rotation angle of $37^{\circ}$ (left) and $60^{\circ}$ (right).

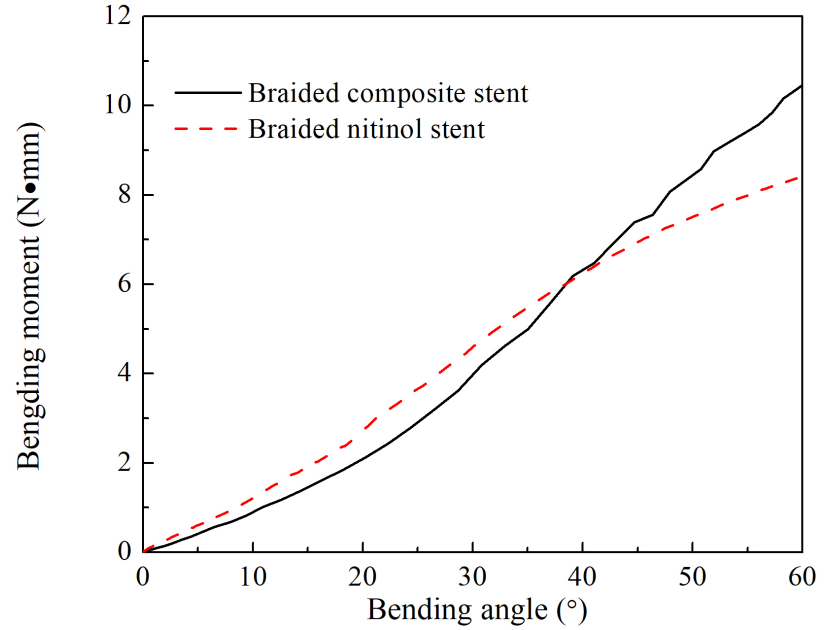

Figure 7: The bending behavior of braided composite stent and braided nitinol stent

The longitudinal flexibility was required for a smooth maneuver through torturous arteries as well as implant inside a curved artery. Enhanced flexibility of stent was also associated with the decreased risk of kinking and incidence of limb thrombosis in tortuous anatomy $[6,7]$. The
BCS desired a larger bending moment when the bending angle was larger than $37^{\circ}$. This could be explained by the larger radial strength of the BCS [9] for larger deformation.

In the present work, we focused on the role of PET strips and the interaction between the PET strips and the NiTi wires on the novel BCS performance. It should be noted that this work does not completely reflect the optimal attributes of the BCS stent, which need consider more parameters, such as the mass fraction of the wires, the braided angle, diameter of the NiTi wires, strip thickness and material properties, etc. The interaction between stent and a torturous artery could provide more complete information on the assessment of this novel stent design. Despite these limitations, the present work demonstrated the potential of the BCS as a substitute for a covered stent with improved mechanical behaviors, which might have significant clinical implications for in-stent restenosis [20]. 
Acknowledgement: The support from the National Science Foundation CAREER award under grant No. 1254095 and the National Natural Science Foundation of China (No. 11372208, No. 11672199) was acknowledged.

\section{References}

[1] Leon L.R. Jr, Dieter R.S., Gadd C.L., Ranellone E., Mills J.L. Sr, Montero-Baker M.F., Gruessner A.C., Pacanowski J.P. Jr, Preliminary results of the initial United States experience with the Supera woven nitinol stent in the popliteal artery, J. Vasc. Surg., 2013, 57, 1014-1022.

[2] Zhao S., Liu X.C., Gu L., The impact of wire stent fabrication technique on the performance of stent placement, J. Med. Dev., 2012, 6, 011007.

[3] Watson T., Webster M.W., Ormiston J.A., Ruygrok P.N., Stewart J.T., Long and short of optimal stent design, Open Heart, 2017, 4 , e000680.

[4] Lee J.H., Krishna S.G., Singh A., Ladha H.S., Slack R.S., Ramireddy S., Raju G.S., Davila M., Ross W.A., Comparison of the utility of covered metal stents versus uncovered metal stents in the management of malignant biliary strictures in 749 patients, Gastrointest. Endosc., 2013, 78, 312-324.

[5] Al-Mukhaini M., Panduranga P., Sulaiman K., Riyami A.A., Deeb M., Riyami M.B., Coronary perforation and covered stents: an update and review, Heart views: Offic. J. Gulf Heart Assoc., 2011, 12, 63.

[6] Rieder E., Dunst C., Martinec D., Cassera M., Swanstrom L., Endoscopic suture fixation of gastrointestinal stents: proof of biomechanical principles and early clinical experience, Endoscopy, 2012, 44, 1121-1126.

[7] Carroccio A., Faries P.L., Morrissey N.J., Teodorescu V., Burks J.A., Gravereaux E.C., Hollier L.H., Marin M.L., Predicting iliac limb occlusions after bifurcated aortic stent grafting: anatomic and device-related causes, J. Vasc. Surg., 2002, 36, 679-684.

[8] Weale A., Balasubramaniam K., Hardman J., Horrocks M., Use of the Aorfix stent graft in patients with tortuous iliac anatomy, J. Cardiovasc. Surg., 2010, 51, 461-466.

[9] Zou Q., Xue W., Lin J., Fu Y., Guan G., Wang F., Wang L., Mechanical characteristics of novel polyester/NiTi wires braided composite stent for the medical application, Results Phys., 2016, 6, 440446.
[10] Kim J.H., Kang T.J., Yu W.-R., Mechanical modeling of selfexpandable stent fabricated using braiding technology, J. Biomech., 2008, 41, 3202-3212.

[11] De Beule M., Van Cauter S., Mortier P., Van Loo D., Van Impe R., Verdonck P., Verhegghe B., Virtual optimization of selfexpandable braided wire stents, Med. Eng. Phys., 2009, 31, 448453.

[12] Guan Y., Lin J., Dong Z., Wang L., Comparative Study of the Effect of Structural Parameters on the Flexibility of Endovascular Stent Grafts, Adv. Mat. Sci. Eng., 2018, 2018.

[13] Rebelo N., Gong X.-Y., Hall A., Pelton A.R., Duerig T.W., Finite element analysis on the cyclic properties of superelastic Nitinol, Proc. Int. Conf. Shape Memory Superelast. Technol., 2006, 157163.

[14] Puskas J.E., Chen Y., Biomedical application of commercial polymers and novel polyisobutylene-based thermoplastic elastomers for soft tissue replacement, Biomacromolecules, 2004, 5, 11411154.

[15] Liu G., Hu H., Zhang P., Wang W., Radial compressive properties of the biodegradable braided regeneration tubes for peripheral nerve repair, J. Industr. Textil., 2006, 36, 35-46.

[16] Balasubramaniam K., Hardman J., Horrocks M., Bulbulia R., The advantages of Aorfix for endovascular repair of abdominal aortic aneurysm, J. Cardiovasc. Surg. (Torino), 2009, 50, 139-143.

[17] Isayama H., Nakai Y., Toyokawa Y., Togawa O., Gon C., Ito Y., Yashima Y., Yagioka H., Kogure H., Sasaki T., Measurement of radial and axial forces of biliary self-expandable metallic stents, Gastrointest. Endosc., 2009, 70, 37-44.

[18] Matsumoto T., Inoue K., Tanaka S., Aoyagi Y., Matsubara Y., Matsuda D., Yoshiya K., Yoshiga R., Ohkusa T., Maehara Y., Radial forces of stents used in thoracic endovascular aortic repair and bare self-expanding nitinol stents measured ex vivo-Rapid res cue for obstruction of the innominate artery using bare selfexpanding nitinol stents, Vascular, 2017, 25, 36-41.

[19] Voúte M.T., Hendriks J.M., Van Laanen J.H., Pattynama P.M., Muhs B.E., Poldermans D., Verhagen H.J., Radial force measurements in carotid stents: influence of stent design and length of the lesion, J. Vasc. Interv. Radiol., 2011, 22, 661-666.

[20] Cicha I., Lyer S., Alexiou C., Garlichs C.D., Nanomedicine in diagnostics and therapy of cardiovascular diseases: beyond atherosclerotic plaque imaging, Nanotechnology Reviews, 2013, 2, 449-472. 